\section{ITC $3 / 49$}

Information Technology and Control

Vol. 49 / No. 3 / 2020

pp. 335-345

DOI 10.5755/j01.itc.49.3.25507
Multichannel Correlation Clustering Target Detection

Received 2020/03/16

Accepted after revision 2020/06/10

HOW TO CITE: Xu, Y., Dong, J., Han, Z., Wang, P. (2020). A Multichannel Correlation Clustering Target Detection. Information Technology and Control, 49(3), 335-345. https://doi.org/10.5755/j01.itc.49.3.25507

\title{
Multichannel Correlation Clustering Target Detection
}

\section{Yan Xu}

College of Physics Science \& Technology, Hebei University, Baoding, China;

Army Engineering University, Shijiazhuang, China

\section{Jiangtao Dong}

The 54th Research Institute of China Electronics Science and Technology Corporation, Shijiazhuang, China

\section{Zishuo Han}

Army Engineering University, Shijiazhuang, China

\section{Peiguang Wang*}

College of Electronic Information Engineering, Hebei University, Baoding; e-mail: pgwang@hbu.edu.cn

\section{*Corresponding author: pgwang@hbu.edu.cn}

During target tracking, certain multi-modal background scenes are unsuitable for off-line training model. To solve this problem, based on the Gaussian mixture model and considering time correlation between pixels, a method that combines the random sampling operator and neighborhood space propagation theory is proposed to simplify the model update process. To accelerate the model convergence, the parameters update strategy based on observation vector proposed in this paper. Finally, a three channel-multimodal background model fusing the HSI color space and gradient information is established in this study. Combining with simplify update theory, the algorithm execution efficiency of HSG-GMM with three channels can reach about $0.05 \mathrm{~s}$ perframe. Experiments indicate that the algorithm has good detection performance when inhibiting ghosts, dynamic background, and shade; thus, the execution efficiency can meet the needs of real-time computing.

KEYWORDS:Mixed Gaussian; random sub-sampling; neighborhood correlation; multi-channel. 


\section{Introduction}

Moving target detection and tracking are affected by scene transformation, occlusion, and noise, and a variety of techniques have introduced to solve such problems. Zhou [23] proposed a multi-level features extraction for discontinuous target tracking and matched the features with Gaussian weight function. Somayyeh [15] completed the target tracking by combined the $\mathrm{PF}$ and genetis algorithm together. Unfortunately, even in CVPR2015-2018, most target tracking algorithms are based on target detection; hence, model training requires a large amount of offline labeled data. With the noise in the multi-modal video environment, the characters of moving targets may irregularly change; thus, a more stable and robust online moving target detection algorithm may be required.

The current primary moving target detection algorithm contains the following: optical flow method [1, 12-13], inter-frame difference method, and background subtraction method [19-20, 24]. The factors such as noise and multi-light source in the optical flow method would have a serious impact on the calculation of the optical flow field distribution. The inter-frame difference method can achieve better results for simple background, but it is sensitive to environmental noise. The background subtraction method achieves accurate detection and high precision, and this type of method is widely utilized. The background is modeled based on the video sequences, and the parameters of the model are used to approximate the pixel value extension of the background. Thus, the performance of this method significantly depends on the accuracy of background models. There is uncertainty present in actual complex scenes, such as dynamic backgrounds and lighting changes [2], and these factors pose a great challenge to accurate background modeling.

Scholars have performed much research on background modeling methods [20, 10-11]. Lai [12] proposed the Gaussian mixture model (GMM) using a mixed Gaussian distribution model to characterize the pixel points in the image frame and obtains the background model through online learning and training. $\mathrm{Li}$ [14] proposed a single-pixel time-series-histogram is used to model the background. The foreground target area is cleaner and has fewer error pixels; however, the method has a large storage overhead and the background generation is slow. Zhu [25] proposed a three-frame difference-based learning rate self-adaption method to update the parameters of the mixed Gaussian background model, to reduce misdetection in the light mutation and target fast motion scenes. Chen [14] introduced the guided filtering, updating method with spaced neighborhood and the space-time smoothing method to mitigate false target moving detection.

The ViBe algorithm [3] introduced random clustering, and employed a random strategy to update the sample pixels, hence avoiding building a model with big data. Ge [16] completed the infrared objects classification using Kullback-Leibler divergence of Gaussian distribution.

However, almost all of these methods have the following shortcomings:

1 When a moving target exists in the initial frame, the detection result may produce a "ghost" reflection.

2 The pixel-by-pixel calculation operation ignores the correlation between pixels, so the operational efficiency is low.

3 The threshold or parameter is fixed during the sample or model update process, resulting in a large difference in terms of target detection accuracy in different scenarios.

In this paper, a moving target is detected using an improved GMM, and the initial parameters of the model are obtained via the inter-frame difference method. A calculation method for the neighborhood correlation is proposed to update the parameters of the Gaussian model. The single pixel feature is combined with the neighborhood random correlation, and the execution efficiency is accounted for when establishing the multimodal background model. The active degree of the pixel is introduced to adjust the learning rate and to accelerate the convergence of the model. Finally, the color-gradient method is adopted to complete the multi-channel Gaussian mixture model in order to help achieve moving target detection. 


\section{Improved GMM Parameter Update Method}

\subsection{Parameter Initialization Method Based on the Spatial Neighborhood of Five-Frame Difference}

To achieve parameter initialization of the mixed Gaussian model, the method combined five-frame difference and intra-frame neighborhood averaging as proposed in this paper.

First, five consecutive frames of images are extracted using the intermediate frame to perform differential operations on the two frames. The difference result is binarized and then subjected to an AND operation to detect whether the current frame contains target information. After removing the motion region from the background image, the five-frame mean value of the corresponding position is calculated as the initial mean value. Meanwhile, an accumulation matrix CA is proposed to record the number of neighboring pixel points:

$$
C A(x, y)=C A(x, y)+1, \quad|f(x, y)-\mu(x, y)|<\alpha \lambda .
$$

Mean matrix and standard deviation are updated as follows:

$$
\begin{aligned}
& \mu(x, y)=(f(x, y)+\mu(x, y)) / 2, \quad|f(x, y)-\mu(x, y)|<\alpha \lambda \\
& \sigma=\sqrt{\frac{1}{5} \sum_{t=1}^{5}\left(f_{t}(x, y)-\mu(x, y)\right)}
\end{aligned} .
$$

Additionally, one of the Gaussian distributions in the mixed Gaussian model is constructed:

$$
\left\{\begin{array}{l}
\mu_{1}(x, y)=\mu(x, y) \\
\sigma_{1}(x, y)=\sigma(x, y) . \\
\varpi_{1}(x, y)=\varpi_{M}
\end{array}\right.
$$

The parameters of the other Gaussian models are obtained as follows:

$$
\left\{\begin{array}{l}
\mu_{k}(x, y)=f(x, y) \\
\sigma_{k}(x, y)=\sigma_{M} \\
\varpi_{k}(x, y)=\varepsilon
\end{array} \quad k=2 . . K .\right.
$$

\subsection{Model Parameter Updating Strategy Combining Random Subsampling and Neighborhood Spatial Propagation Theory}

Considering the continuity of image spatial information, mutual difference and similarity exist between pixels [3]. The mutual difference can explore the variation of pixel points. The similarity between neighborhoods can reduce redundant information between pixels [13], and simultaneously avoid repeating modeling with similarities. Therefore, the adjacent pixel correlation theory is proposed to measure the correlation of pixels in the neighborhood of the GMM parameter updating process. For the neighborhood of pixels with the same category, the parameter updating calculation of the Gaussian distribution is not performed. Generally, the pixel correlation of the background image is high, and the computation of parameter updating based on neighborhood pixel correlation metrics can greatly decrease.

$x_{i}, y_{i}$ are the gray values for two adjacent pixels in the $\mathrm{i}^{\text {th }}$ frame of the sequence images in sliding window $\mathrm{N}$ at time $t$ (showed in Figure1).

\section{Figure 1}

Sequence of images in sliding window $\mathrm{N}$ at time $\mathrm{t}$

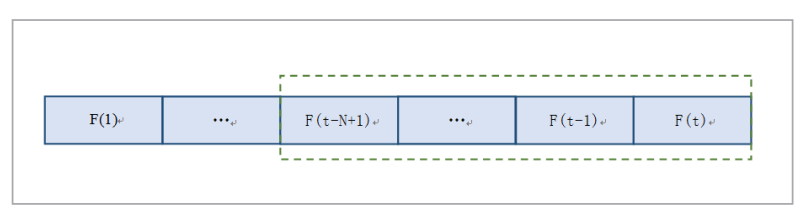

$D\left(x_{t}\right)=\frac{1}{N} \sum_{i=1}^{N}\left(x_{i}-E\left(x_{t}\right)\right)^{2}$ and $D\left(y_{t}\right)=\frac{1}{N} \sum_{i=1}^{N}\left(y_{i}-E\left(y_{t}\right)\right)^{2}$ is the variance of $x_{i}, y_{i}, \operatorname{cov}\left(x_{t}, y_{t}\right)=\frac{1}{N} \sum_{i=1}^{N}\left(x_{i}-E\left(x_{t}\right)\right)$ $\left(y_{i}-E\left(y_{t}\right)\right)$ is the covariance of the two pixels, $E\left(x_{t}\right)=\frac{1}{N} \sum_{i=1}^{N} x_{i}$ and $E\left(y_{t}\right)=\frac{1}{N} \sum_{i=1}^{N} y_{i}$ is the mean value and $N$ is the number of frames participating in the neighborhood correlation calculation of the image sequence.

The concept of the sliding window [1] is introduced herein to estimate the real-time mean and variance value online.

To calculate the neighborhood correlation for any time $t$, the former $t-N+1$ frame image is essential. To simplify calculations and reduce storage space, $E\left(x_{t}\right)$ 
and $D\left(x_{t}\right)$ are simplified and replaced by the weighted average of the mean and variance value of GMM:

$$
\begin{aligned}
& \hat{E}\left(x_{t}\right)=\sum_{k=1}^{K} \omega_{k, t} \mu_{k, t-1}, \\
& \hat{D}\left(x_{t}\right)=\sum_{k=1}^{K} \omega_{k, t} \sigma_{k, t-1}
\end{aligned}
$$

where $\mathrm{K}$ is the number of Gaussian distributions, and the cross-correlation function is

$$
\operatorname{co\hat {v}}\left(x_{t}, y_{t}\right)=\frac{1}{N} \sum_{i=1}^{N}\left(x_{i}-\hat{E}\left(x_{t}\right)\right)\left(y_{i}-E\left(y_{t}\right)\right) .
$$

(General sliding window N=50). The neighborhood pixel correlation is

$$
\hat{\gamma}_{x y}=\frac{\operatorname{cov}\left(x_{t}, y_{t}\right)}{\sqrt{\hat{D}\left(x_{t}\right)} \sqrt{D\left(y_{t}\right)}} .
$$

The current pixel correlation in 8-neighborhood is calculated to update the GMM parameters using Eq. 7. When the pixel correlation value exceeds a certain threshold $T_{\gamma}$, the neighborhood pixel can directly be divided into the same Gaussian model with the current pixel, thereby avoiding duplicated computations. Otherwise, for the pixels that do not belong to the same category, the model update strategy is still adopted. Such an update strategy utilizes the spatial propagation characteristics of pixel values, and the background model gradually spreads to the neighborhood, which is beneficial for speeding up the recognition of the ghost region.

In the process of practical calculation, the image block update approach was implemented for the current image. Only the GMM parameters of the center point in the window of a $3 \times 3$ neighborhood could be updated firstly, then centered on the current point, the pixel correlation $\hat{\gamma}_{x y}$ in 8-neighborhood is calculated, after normalization correlation processing relative to the center point, the normalized correlation distance between each neighborhood point and the center point is

$$
\hat{\gamma}_{x y}^{\prime}=\frac{\left|\hat{\gamma}_{x y}-\gamma_{x x}\right|}{\hat{\gamma}_{x x}},
$$

when the correlation distance $\hat{\gamma}^{\prime}$ is less than the threshold $T_{\gamma}^{\prime}$ (typically set to 0.25), the neighborhood pixel can directly be divided into the same Gaussian model with the current pixel. Otherwise, for the pixels that do not belong to the same category, the model update strategy is still adopted.

In the meantime, Ge [16] noted that when the model of pixel change cannot be accurately judged, the random update strategy can partly simulate the uncertainty variation. Owing to the slow update of the background in the image, even if there is a dynamic background, the background will not significantly change between adjacent frames. Therefore, updating the pixels determined as the background frame by frame is unnecessary. For this, a random sub-sampling strategy is proposed in this paper to select the background pixels and update model parameters: the pixels that have been identified as background are randomly selected by $1 / \varphi$ to participate in the model update calculations, combined with the neighborhood correlation update strategy, the neighborhood spatial propagation characteristics are used to make model copies with the neighborhood of these update points.

Random subsampling and neighborhood correlation blocks update strategy combined the spatial and sequential correlation and variability together, and therefore updating frequency of the background pixels can be slashed by at least an order of magnitude.

\section{Figure 2}

Model parameter update process

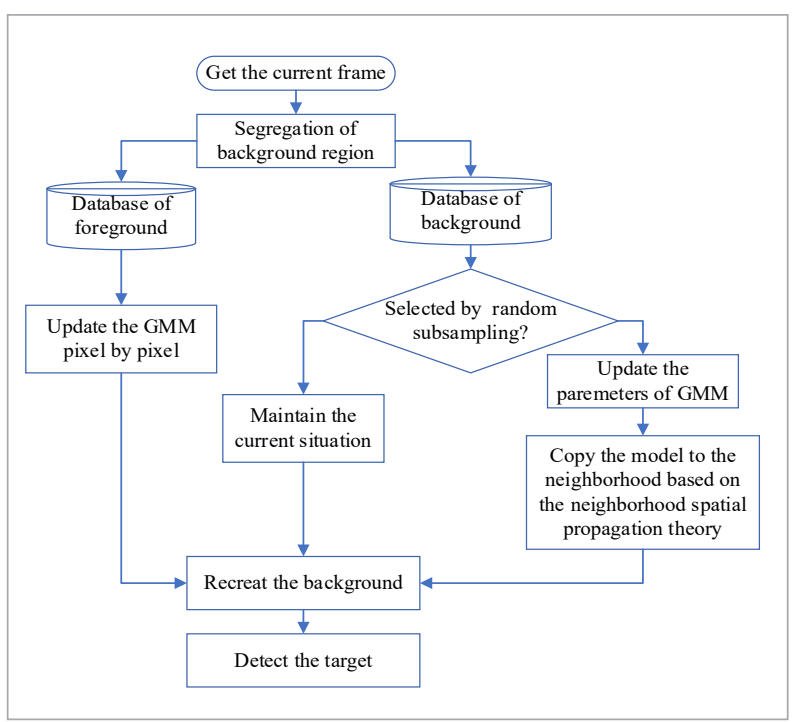




\subsection{Parameters Update Strategy Based on Observation Vector}

In the basic GMM algorithm, the parameters of the Gaussian model can be updated as:

$$
\begin{aligned}
& \varpi_{k, t}=(1-\alpha) \times \varpi_{k, t-1}+\alpha \varpi_{k, t-1} \\
& \mu_{k, t}=\left(1-\beta_{k}\left(x_{t}\right)\right) \times \mu_{k, t-1}+\beta_{k}\left(x_{t}\right) \times x_{t} \\
& \sigma_{k, t}^{2}=\left(1-\beta_{k}\left(x_{t}\right)\right) \times \sigma_{k, t-1}^{2}+\beta_{k}\left(x_{t}\right) \times\left(x_{t}-\mu_{k, t-1}\right)^{2},
\end{aligned}
$$

where $\varpi_{k, t}$ is the weight of kth Gaussian model. $\alpha$ is the learning rate. $\mu_{k, t}$ is the mean value of the $k$ th Gaussian model. $\sigma_{k, t}^{2}$ is the variance of the $k$ th Gaussian model. $\beta_{k}\left(x_{t}\right)=\alpha \times p_{k}\left(x_{t}\right)$, where $p_{k}\left(x_{t}\right)$ is the probability value of the $k$ th Gaussian model at the point $x_{t}$.

During the update processing, the models that are matched can update all the parameters using Eq. 9, other models that are not matched can only update the weights, and this may slow down the GMM update speed. It may be assumed that the $k$ th Gaussian distribution does not match at the first $\mathrm{n}$ frames, and the weight is updated to $\varpi_{k, t}=(1-\alpha)^{n} \times \varpi_{k, 0}$, where $\alpha$ is usually small, which makes the reduction rate of $\varpi_{k, t}$ slower; however, this distribution should not conform to the background model, and such a distribution may achieve faster convergence.

A count vector with $K$ elements is introduced in this paper, where $c_{k}$ records the observations of the $k$ th Gaussian distribution.

The posterior probability that the pixel matches the $k$ th Gaussian distribution is calculated as

$$
q_{k}\left(x_{t}\right)=p_{k}\left(x_{t}\right) / \sum_{j=1}^{K} p_{j}\left(x_{t}\right)
$$

Making $\quad c_{k}\left(x_{t}\right)=c_{k}\left(x_{t-1}\right)+q_{k}\left(x_{t}\right), c_{k}(0)=0, \quad$ using $\hat{\eta}\left(x_{t}\right)=q_{k}\left(x_{t}\right)\left(\frac{1-\alpha}{c_{k}\left(x_{t}\right)}+\alpha\right)$ to replace $\beta_{k}\left(x_{t}\right)$, and the new formulas for updating the mean and variance is

$\mu_{k, t}=\left(1-\hat{\eta}\left(x_{t}\right)\right) \times \mu_{k, t-1}+\eta\left(x_{t}\right) \times x_{t}$

$\sigma_{k, t}^{2}=\left(1-\hat{\eta}\left(x_{t}\right)\right) \times \sigma_{k, t-1}^{2}+\eta\left(x_{t}\right) \times\left(x_{t}-\mu_{k, t-1}\right)^{2}$

where $\varpi_{k, t}=(1-\alpha) \times \varpi_{k, t-1}+\alpha q_{k}\left(x_{t}\right)$. If the pixel does not match successfully, $\sum_{j=1}^{K} p_{j}\left(x_{t}\right)=0$, then all Gaussian components are degraded.

\section{Target Detection Based on Color-Gradient Method}

The pixel information cannot be fully utilized when establishing the mixed Gaussian model based solely on gradation modeling, and the detection result may generate many hollow regions. Maha [16] produced a multi-information fusion method to achieve the description of the targets. A background modeling method based on color information is derived [17, 21]. Nevertheless, RGB color space mixes the color and brightness information together, and when the brightness changes, the values of the three-color channels will all change, which reduces the color discrimination ability. In contrast, in an HSI space, luminance is relatively independent, and Jiang [9] verifies that the performance of HSI is superior to that of RGB in target detection. The color has good invariance for target shape, scale, etc., and gradient has good invariance for illumination. According to this, color and gradient features are fused in this paper to establish a three-channel-GMM method to complete the background model and then detect the target.

First, the gradient is obtained from the original gray image. Second, the hue and saturation features are extracted in the HSI space. The background model is then initialized and trained separately for the hue, saturation, and gradient features. Finally, the Hue, Saturation, Gradient (HSG) channels are obtained. The mixed Gaussian modeling of the three channels can present the background in detail. Detection of the moving targets is completed. If the HSI value of the current pixel matches any Gaussian distribution in one channel, the point is divided into the background; otherwise, it is identified as the motion foreground. Herein, this three-channel based GMM algorithm is called HSG_GMM.

To verify the sophistication of the new algorithm, the traditional GMM, RGB_GMM algorithm, ViBe algorithm, and the HSG_GMM algorithm that is proposed herein were executed in the same hardware panel: Intel Core i5-5300U CPU, 8G RAM; the frame size is $352 \times 228$, and the results are shown in Figure 3. 
Figure 3

Algorithm execution efficiency comparison

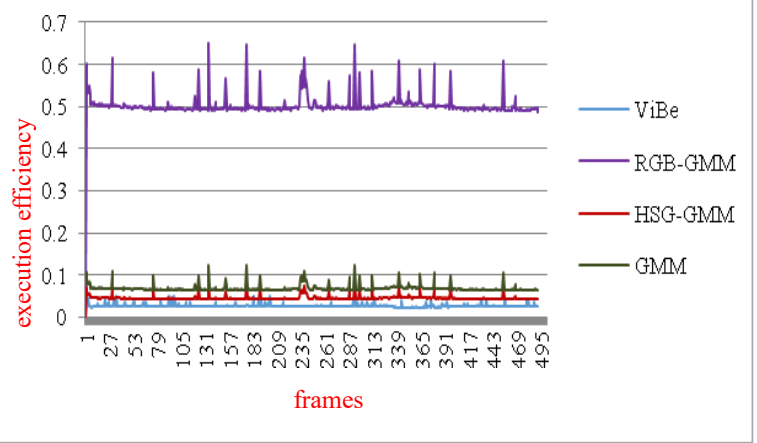

The average execution efficiency of ViBe is about $0.04 \mathrm{~s}$ per-frame, and therefore it can be used for real-time target detection. The mean value of GMM is approximately $0.0 \% \mathrm{~s}$, which is suitable for non-strict demands for real-time execution. RGB-GMM has the lowest efficiency (about 0.5s per-frame) and can only be used for off-line detection. HSG-GMM proposed in this paper adopts a three-channel Gaussian modeling method although it increases the number of channels. The introduction of a combination of random sub-sample and neighborhood correlation metric measurements can decrease the final total computation (about 0.05s per-frame). Although the actual running time cannot be compared with that of ViBe, it can still achieve real-time processing while meeting accuracy requirements.

\section{Experiment and Analysis}

\subsection{Performance Analysis}

The experiment was conducted using the LASIESTA Database [6] of Grupo de Tratamiento de Imagenes of Universidad Politecica de Madrid (UPM). And compare the results achieved by HSG_GMM algorithm proposed in this paper with results achieved by other methods (which contained the traditional GMM [12], RGB_GMM [20] and ViBe [18]) on the same image dataset.

The performance of the algorithm is evaluated in terms of accuracy and precision [8]. The data in "O_
CL_01" are used to calculate the P and A of the GMM, RGB_GMM, and HSG_GMM algorithms.

Of these frames in the O_CL_01 dataset, moving targets with tree occlusion are found in frames 135-206. In Figure 5, the different algorithms were found to obtain almost the same detection result for static background scene; however, their accuracy and precision were different for the moving targets. Compared with the RGB-GMM algorithm, the HSG-GMM algorithm improves the accuracy and precision in some interference images. The basic principle of the ViBe algorithm is different from the other three algorithms and therefore, the distribution of the detection results is also different: ViBe can obtain highly accurate results in certain regions but it fluctuates in a wide range in the moving target area.

\section{Figure 4}

Performance of the algorithm in verifying the results: (a) precision; (b) accuracy

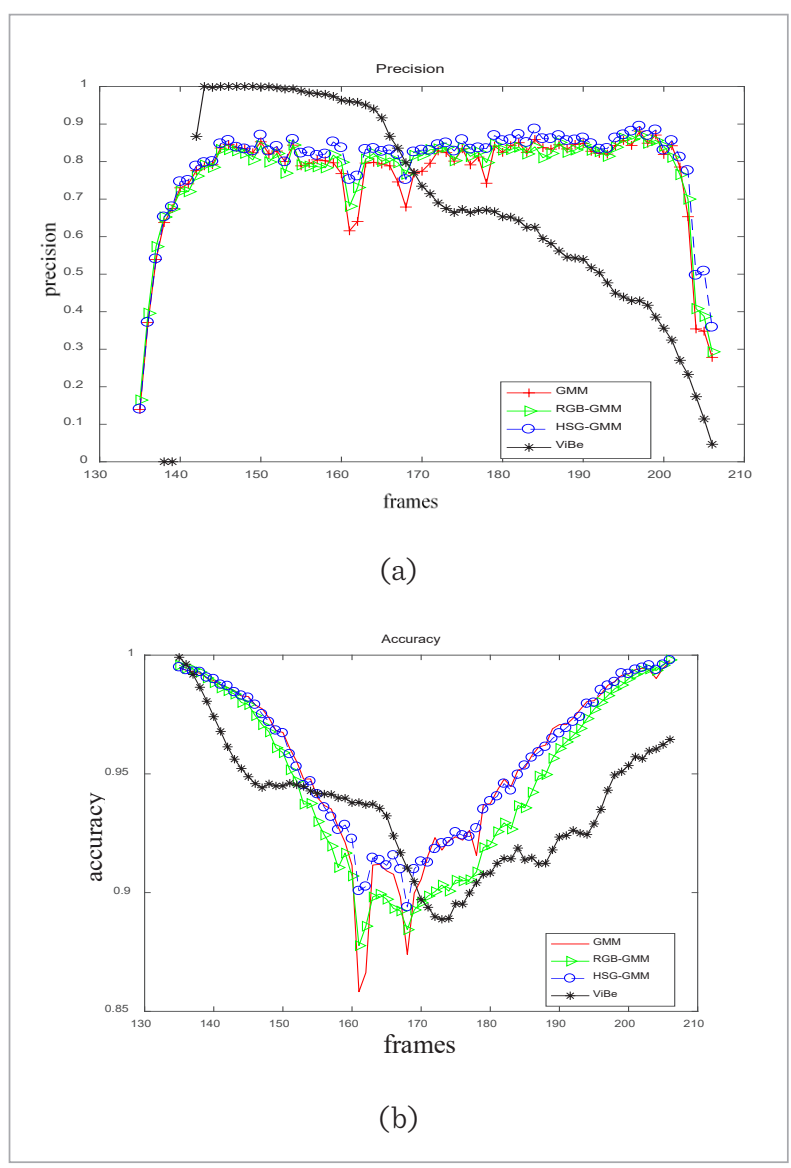


Figure 5

Result of frame 161 of O_CL_01 data set: (a) original image; (b) true value image; (c) GMM result; (d) RGB_GMM result; (e) ViBe result; (f) HSG_GMM result

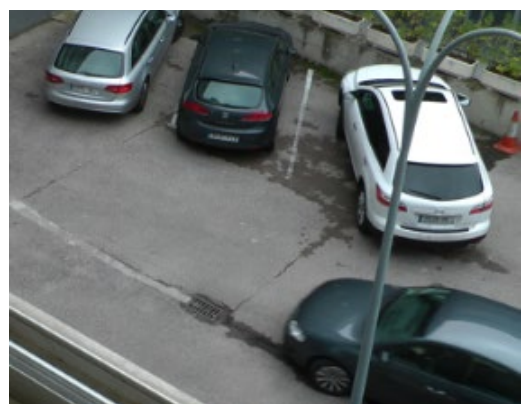

(a)

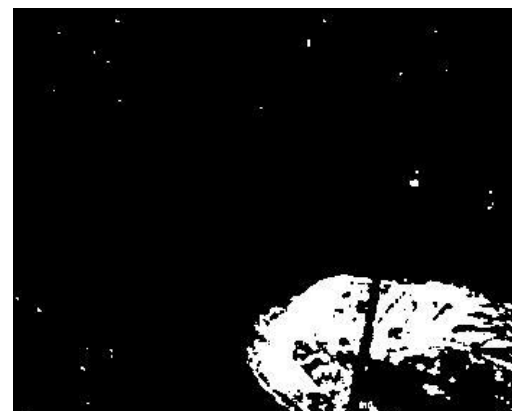

(d)

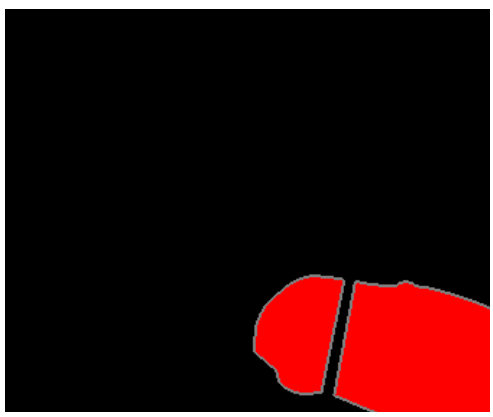

(b)

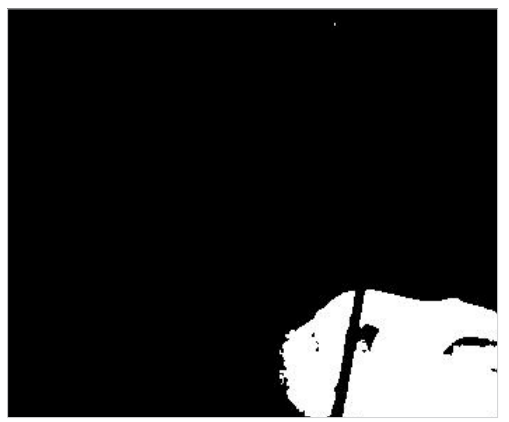

(e)

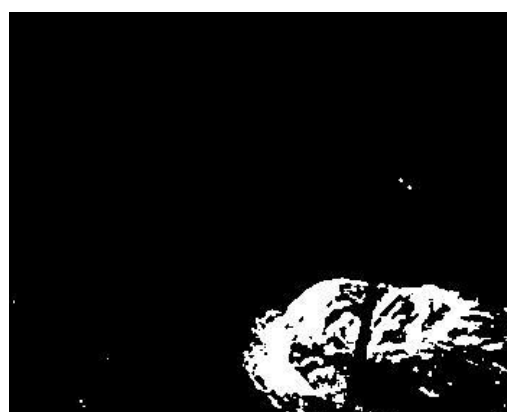

(c)

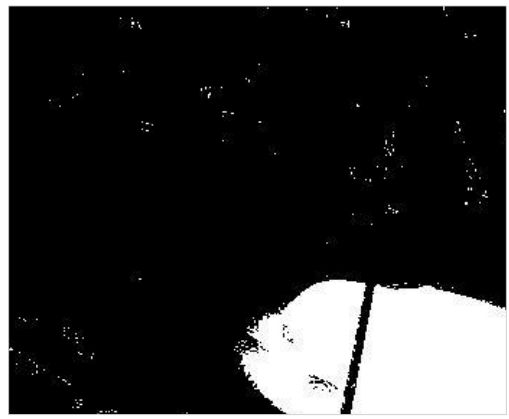

(f)
Figure 5 shows the detection result for the 161st frame. For the detection of pure background without moving target, the precision values of different algorithms are identical in addition to some random noise. However, for the moving target detection, GMM and RGB_GMM could generate more holes, and ViBe can inhibit background noise, but the holes in the target still remain.

\subsection{Effect Comparison}

\subsubsection{Static Background Test}

The video sequence "singnal.avi” is used to verify the effectiveness when there is a moving target in the initial static background.
Figure 6 showed the results of four different algorithms in detecting the $6^{\text {th }} 14^{\text {th }}$ and $58^{\text {th }}$ frames in the video sequence "singnal.avi". The "ghost reflection" caused by the initial motion target in the basic GMM algorithm and the ViBe algorithm is well suppressed in the proposed algorithm. For the influence of the color feature, the RGB_GMM retained more noise in the scene. The detected moving target is more complete, and the noise interference caused by the instability of the RGB value is overcome by the HSG_GMM algorithm. The update strategy utilizes the spatial propagation characteristics of pixel values speeds up the recognition efficiency, and the ghost reflection can be retrained obviously, but other erroneous judgement in the boundary has been introduced, and this made the edge of the target vague. 


\section{Figure 6}

Detection result of moving target in initial video in static background: (a) Initial image; (b) GMM result; (c) RGB_GMM result; (d) ViBe result; (e) HSG_GMM result

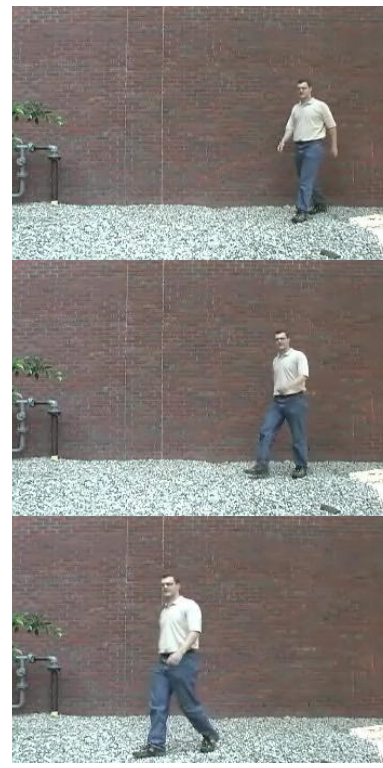

(a)

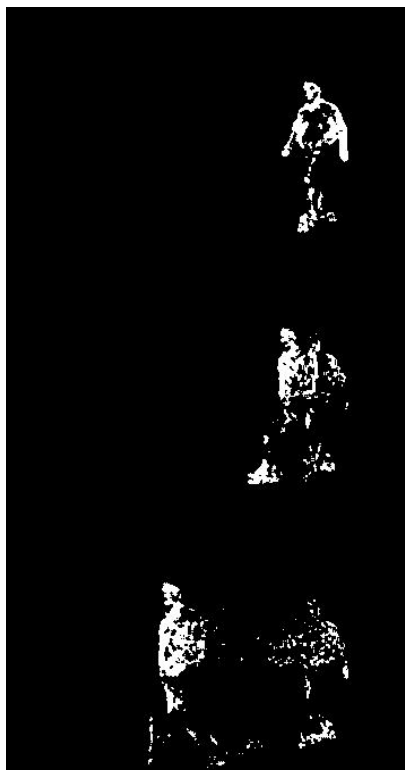

(b)

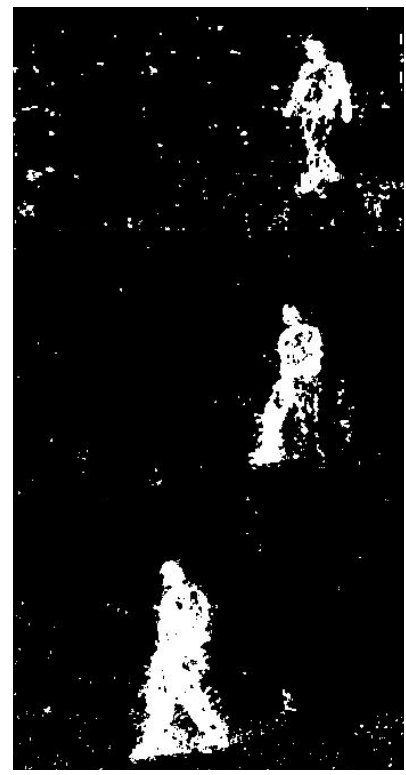

(c)

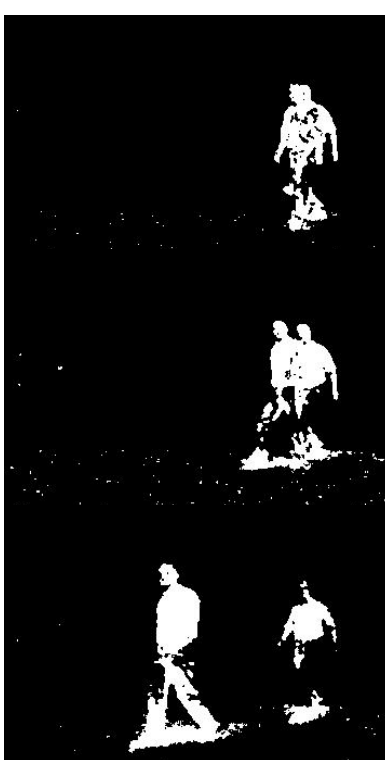

(d)

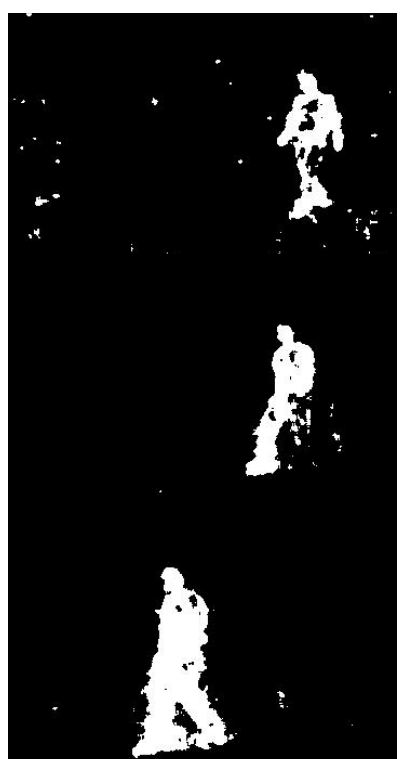

(e)

\subsubsection{Background Test Complex}

Figure 7 shows the detection result of the moving target in the snow scene of the dataset "O_SN_01". The image $\mathrm{x}-1$ in each line in Figure'7 showed the initial image of $320^{\text {th }}$ frame in the video and the result under the four different algorithms. The image $x-2$ and $\mathrm{x}-3$ in each line are the results of the $348^{\text {th }}$ frame and the $361^{\text {th }}$ frame. RGB_GMM suppresses the dynamic 


\section{Figure 7}

Moving target detection in a dynamic background environment: (a1)-(a3): initial image; (b1)-(b3): GMM; (c1)-(c3): RGBGMM; (d1)-(d3):ViBe; (e1)-(e3): HSG-GMM

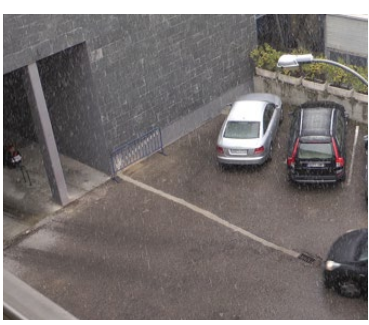

$(\mathrm{a}-1)$

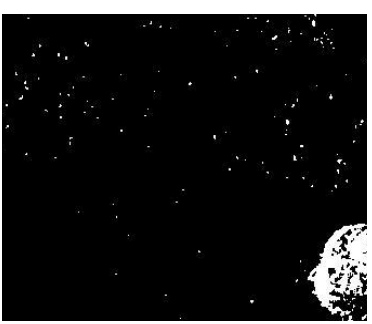

$(b-1)$

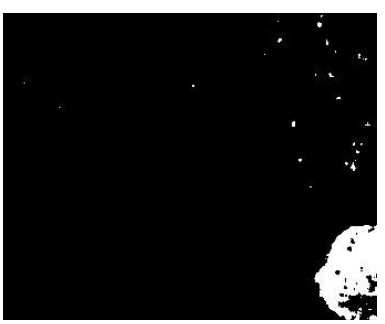

$(\mathrm{c}-1)$

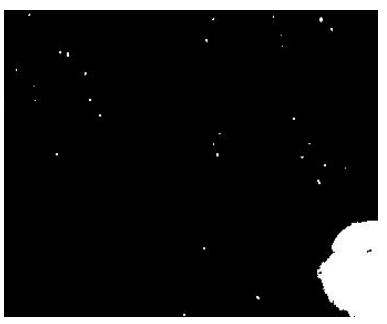

$(\mathrm{d}-1)$

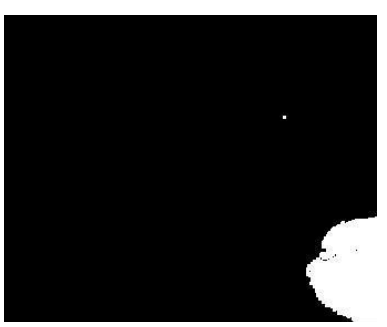

$(\mathrm{e}-1)$

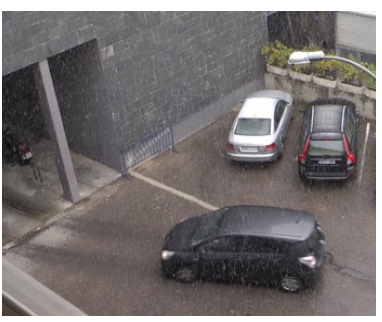

$(\mathrm{a}-2)$

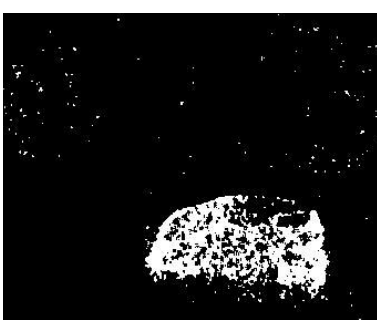

(b-2)

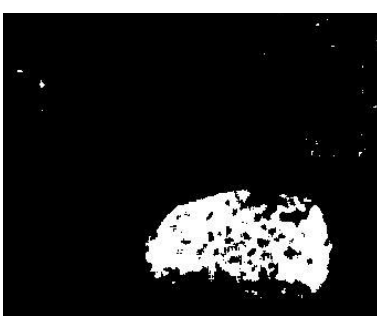

(c-2)

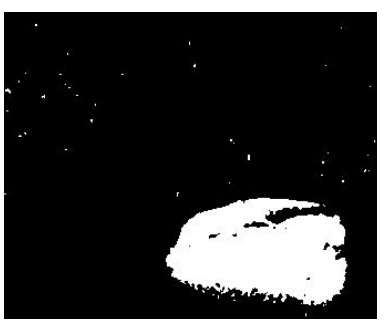

(d-2)

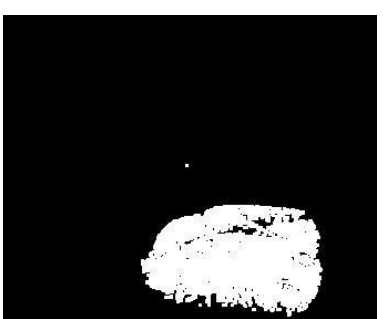

(e-2)

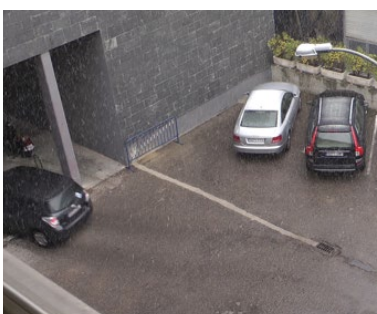

(a-3)

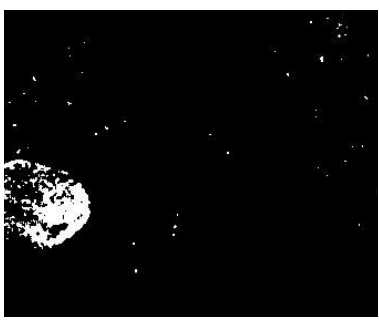

(b-3)

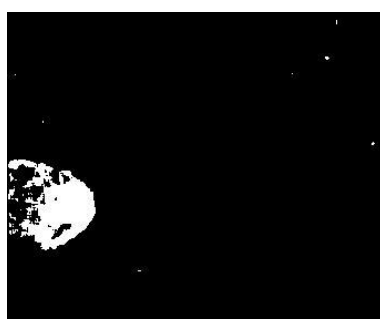

(c-3)

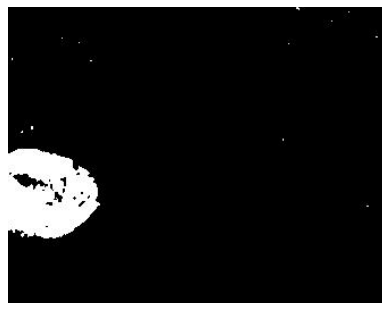

(d-3)

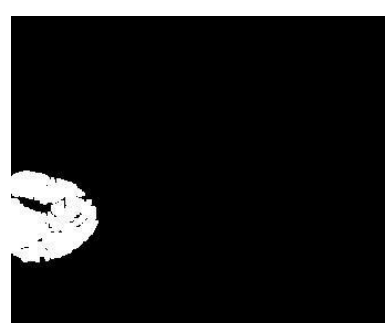

(e-3) 
background of the snowflake to a greater extent than the basic GMM; however, the noise cannot be eliminated absolutely. The HSG-GMM algorithm can essentially eliminate the influence of the complex background, and the detected moving target area is also relatively complete. The observation vector introduced in the HSG-GMM algorithm has an ability enhancement for noise suppression than ViBe, hence the HSG-GMM algorithm obtained a better background model and an integrated target region.

\section{Conclusions}

A parameter initialization method based on the spatial neighborhood of a five-frame difference improved the accuracy of the initial value of the parameters, and the constructed mixed Gaussian model is more in line with the characteristics of the background pixels. The parameters update strategy based on observation vector proposed in this paper speed up the converges of the models, combining with the random subsampling

\section{References}

1. Barron, J. L., Fleet, D. J., Beauchemin, S. S., Burkitt, T. A. Performance of Optical Flow Techniques. Computer Vision and Pattern Recognition, 1994,12, 43-77. https:// doi.org/10.1007/BF01420984

2. Bouwmans, T., El Baf, F., Vachon, B. Background Modeling Using Mixture of Gaussians for Foreground Detection - A Survey. Recent Patents Computer Science, 2008, 1(3), 219-23\%. https://doi. org/10.2174/1874479610801030219

3. Barnich, O., Droogenbroeck, M. V. ViBe: A Universal Background Subtraction Algorithm for Video Sequences. IEEE Transactions on Image Processing, 20(6), 1709-1724. https://doi.org/10.1109/TIP.2010.2101613

4. Chen, Y. Y., Wang, J. Q, Lu, H. Q. Learning Sharable Models for Robust Background Subtraction. IEEE International Conference on Multimedia and Expo, Turin, Italy, 2015. https://doi.org/10.1109/ICME.2015.7177419

5. Choi, M., Sweetman, B. Efficient Calculation of Statistical Moments for Structural Health Monitoring. Structural Health Monitoring, 2010, 9(1), 13-24. https://doi. org/10.1177/1475921709341014

6. Cuevas, C., Yáñez, E. M., García, N. Labeled Dataset for Integral Evaluation of Moving Object Detection Algo- and neighborhood spatial propagation theory, the algorithm execution efficiency of HSG-GMM with three channels can reach about 0.05 s per-frame. Color information of the HSI space is combined with gradient information to construct a multi-channel model establishment and update mechanism, which makes the background model close to perfect.

Experiments show that the proposed algorithm HSGGMM is greatly improved when suppressing the influence of complex backgrounds and detecting target integrity. The influence of the moving target in the initial stage is eliminated and there are fewer target holes; however, some other erroneous judgement in the boundary has been introduced, and this made the edge of the target vague. Furthermore, some failed experiment showed that the HSG-GMM algorithm could not detect the slow-moving targets more clearly under camera motion conditions than ViBe.

\section{Acknowledgement}

The authors acknowledge the National Natural Science Foundation of China (Grant: 11771115).

rithms: LASIESTA. Computer Vision Image Understanding, 2016, 152, 103-117. https://doi.org/10.1016/j. cviu.2016.08.005

7. Ge, H. L., Zhu, Z. Y., Lou, K. Classification of Infrared Objects in Manifold Space Using Kullback-Leibler Divergence of Gaussian Distributions of Image Points. Symmetry, 2020, 12(3), 434. https://doi.org/10.3390/ sym12030434

8. Goyette, N., Jodoin, P. M., Porikli, F., Konrad, J., Ishwar, P. Changedetection.net: A New Change Detection Benchmark Dataset. 2012 IEEE Computer Society Conference on Computer Vision and Pattern Recognition (CVPR), Workshops, Rhode Island, 2012, 1-8. https://doi.org/10.1109/CVPRW.2012.6238919

9. Jiang, Y.S., Ma, J. W. Combination Features and Models for Human Detection. IEEE Conference on Computer Vision and Pattern Recognition (CVPR), Boston, USA, 2015,240.https://doi.org/10.1109/CVPR.2015.7298620

10. Kaewtrakulpong, P., Bowden, R. An Improved Adaptive Background Mixture Model for Realtime Tracking with Shadow Detection. Video-Based Surveillance Systems, Springer, Boston, MA, 2002, 11, 135-144. https://doi. org/10.1007/978-1-4615-0913-4_11 
11. Kang, K., Cao, Y., Zhang, J. Salient object Detection and Classification for Stereoscopic Images. Multimedia Tools App, 2016, 75(3), 1443-145\%. https://doi. org/10.1007/s11042-014-2142-8

12. Lai, L. J., Xu, Z. Y., Zhang, X. Y. An Improved Gradient Optical Flow Method for Image Stabilization System. Infrared Laser Engineering, 2016, 45(4), 273-279. https://doi.org/10.3788/irla201645.0428004

13. Li, W. J, Yao, J. G., Dong, T. Z. Moving Vehicle Detection Based on an Improved Interframe Difference and a Gaussian Mode. 2015 8th International Congress on Image and Signal Processing (CISP), Shenyang, 2015, 969-973. https://doi.org/10.1109/CISP.2015.7408019

14. Li, X. Y., Ma, D. Z., Fu, Y. Moving Object Detection Using Mixed Gauss Background Model Based on Three Frame Differencing. Journal of Jilin University, 2018, 36(4), 61-69.

15. Moghaddasi, S. S., Faraji, N. A Hybrid Algorithm Based on Particle Filter and Genetic Algorithm for Target Tracking. Expert Systems with Applications, 2020, 147(1), 113188. https://doi.org/10.1016/j.eswa.2020.113188

16. Maha, M. A., Shedeed, H. A., Hussein, A. S. A new Technique for Background Modeling and Subtraction for Motion Detection in Real-time Videos. The 17th IEEE International Conference on Image Processing, Hong Kong, China, 2010, 3453. https://doi.org/10.1109/ ICIP.2010.5653748

17. Martin, D., Fahad, S. K., Michael, F. Adaptive color Attributes for Real-time Visual Tracking. IEEE Conference on Computer Vision and Pattern Recognition (CVPR), Columbus, USA, 2014, 1090. http://dx.doi.org/ 10.1109/ CVPR.2014.143

18. Noh, S. J., Jeon, M. A New Framework for Background Subtraction Using Multiple Cues. Asian Conference on
Computer Vision, Daejeon Korea, 2012, 49. https:/doi. org/10.1007/978-3-642-37431-9_38

19. Stauffer, C., Grimson, W. L. Adaptive background Mixture Models for Real-time Tracking. Computer Society Conference on Computer Vision and Pattern Recognition, IEEE Computer Society Press: Washington, DC, USA 1999, 2246.

20. Wren, C. R., Azarbayejani, A., Darrell T. Pfinder: Real-time Tracking of the Human Body. IEEE Transactions on Pattern Analysis and Machine Intelligence, 1997, 19(7), 780-785. https://doi.org/10.1109/34.598236

21. Xu, Y., Dong, J. T., Wang, S. H. Adaptive image Interpolation Algorithm Based on the Fuzzy Logic. Acta Physica Sinica, 2010, 59(11), 7535-7539. http://dx.doi.org/ CNKI:SUN:WLXB.0.2010-11-004.

22. Yandi L., Xu X. P., Wang H. C. Application of RHT Based on Character String Constraint in Ellipse Detection. Chinese Journal of Scientific Instrument, 2017, 38(1), 50-56. http://dx.doi.org/ 10.3969/j.issn.02543087.2017.02.023.

23. Zhou, B., Duan, X. M., Ye, D. J., Wei, W., Wozniak, M., Polap, D., Damaševičius, R. Multi-Level Features Extraction for Discontinuous Target Tracking in Remote Sensing Image Monitoring. Sensors, 2019, 19(22), 4855. https://doi.org/10.3390/s19224855

24. Zhao, X. D., Liu, P., Liu, J. F., Tang, X. Adaptive Background Estimation of Outdoor Illumination Variations for Foreground Detection. Acta Automatica Sinica, 2011, 37(8), 915-922. https://doi.org/10.1109/ VCIP.2011.6115943

25. Zhu, W. J., Wang, G. L., Tian, J. Spatio-temporal Adaptive Mixture of Gaussians for Moving Objects Detection in Complex Background Scenes. Transaction of Beijing Institute of Technology, 2018, 38(2), 165-172. 